\title{
Tips and tricks for getting more out of your delayed primary repair of ruptured flexor pollicis longus tendon
}

\author{
Charles Yuen Yung Loh, Alethea Tan, Makarand Tare \\ St Andrew's Centre for Plastic and Reconstructive Surgery, Broomfield Hospital, Chelmsford CM1 7ET, UK.
}

Correspondence to: Dr. Charles Yuen Yung Loh, St Andrew's Centre for Plastic and Reconstructive Surgery, Broomfield Hospital, Chelmsford CM1 7ET, UK. E-mail: chloh_yy@hotmail.com

How to cite this article: Loh CYY, Tan A, Tare M. Tips and tricks for getting more out of your delayed primary repair of ruptured flexor pollicis longus tendon. Plast Aesthet Res 2017;4:135-6.

Sir,

Primary tendon repairs are often difficult in patients with delayed presentation. Tendons are contracted and shortened with extensive scarring occurring along the path of the tendon. Pulleys and the wound bed can be filled with granulation tissue which obstructs the passage of the tendon. Many of such patients would then be treated with a two stage tendon reconstruction, which involves the insertion of a silicone rod for pseudosheath formation ${ }^{[1]}$ before tendon grafting at a second stage. This however, would set the patient back for roughly six months, especially involving multiple visits to physiotherapy and being off work. We would like to describe several tips and tricks in our armamentarium and illustrate these using a case we recently encountered [Figure 1].

A 40-year-old mechanic presented with a 5-weekold rupture of the flexor pollicis longus (FPL) at the interphalangeal joint (IPJ) of the left thumb. A plan for a two-stage reconstruction of the tendon was discussed with him and was scheduled for a silicone rod insertion. Bruner incisions to zone 3 were made and the distal end of FPL was seen but the proximal end had retracted to the wrist.

Our tips and tricks used were as follows:

1. Use of a size 6 feeding catheter attached to a salinefilled syringe for hydrodissection of the path. Palpation of the catheter through the skin can be performed to allow localisation of path.

2. Adequate debridement of granulation and scar tissue was performed along the path of the tendon, especially under the pulleys of the thumb. Pulleys were also stretched using a fine tooth artery clip for several seconds, which avoided any venting.

3. The FPL tendon found at the wrist level was delivered through a wrist incision and stretched under tension for 2 min using an artery clip. Le Viet's releasing incisions can be performed at the tendon-muscle belly junction for added length if required. ${ }^{[2]}$

4. When attaching the FPL tendon to the feeding catheter, a gap is left when suturing the two together. Careful retraction of the feeding catheter with the tendon is then performed.

5. A combination of a two strand modified Kessler core suture and a mattress suture was used with a $3 / 0$ 


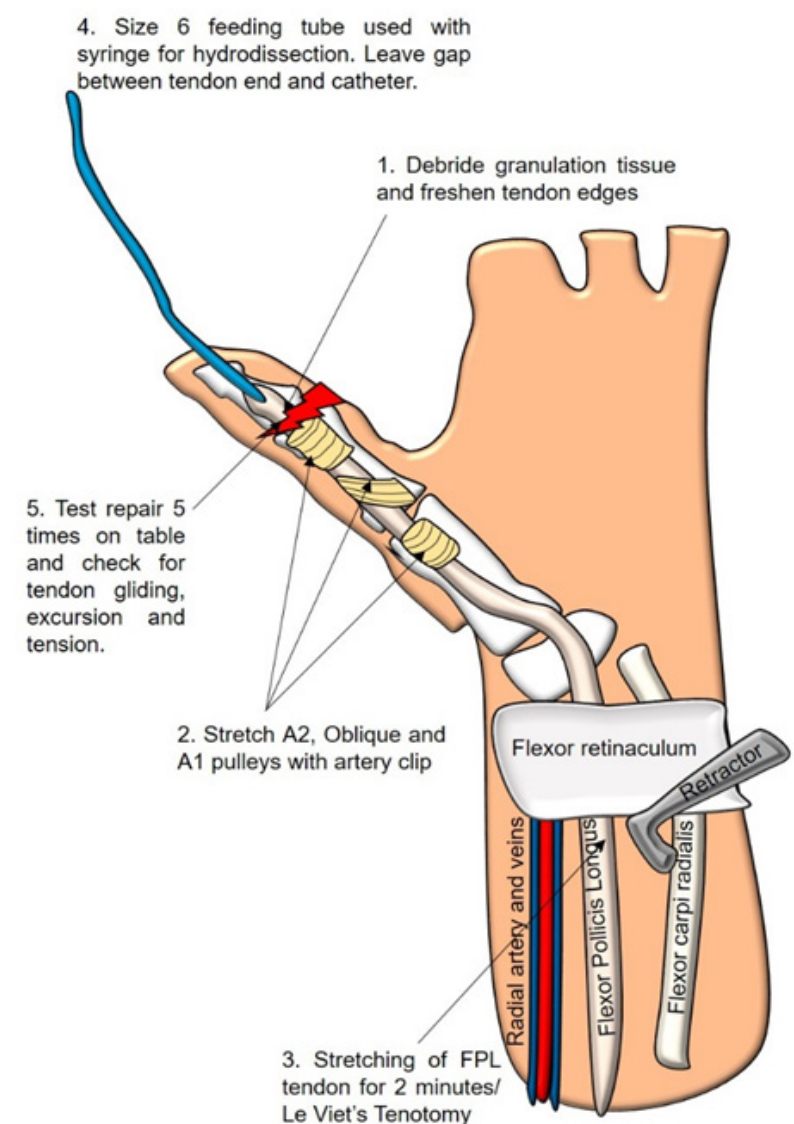

Figure 1: Schematic diagram illustrating the tips and tricks used for getting more out a primary FPL tendon repair. FPL: flexor pollicis longus

prolene suture. A running epitendinous repair with $4 / 0$ prolene was performed.
We tested our repair on table by flexing and extending the thumb IPJ five times, and ensured tendon gliding and excursion was not compromised. The thumb was splinted with a dorsal blocking splint in slight IPJ flexion. If on table tendon rupture or inadequate tendon excursion and gliding occurs despite the techniques performed above, a two-stage tendon reconstruction is then indicated.

\section{DECLARATIONS}

\section{Authors' contributions}

Manuscript preparation: C.Y.Y. Loh, A. Tan

Manuscript review: M. Tare

\section{Financial support and sponsorship}

None.

\section{Conflicts of interest}

There are no conflicts of interest.

\section{Patient consent \\ Obtained.}

\section{Ethics approval}

Not required.

\section{REFERENCES}

1. Bassett CA, Carroll RE. Formation of a tendon sheath by silicone-rod implants. J Bone Joint Surg Am 1963;45:884-5.

2. Le Viet D. Flexor tendon lengthening by tenotomy at the musculotendinous junction. Ann Plast Surg 1986;17:239-46. 